\title{
Corrections
}

\section{Correction: Araujo et al., "Foxp1 in Forebrain Pyramidal Neurons Controls Gene Expression Required for Spatial Learning and Synaptic Plasticity"}

In the article "Foxp1 in Forebrain Pyramidal Neurons Controls Gene Expression Required for Spatial Learning and Synaptic Plasticity" by Daniel J. Araujo, Kazuya Toriumi, Christine O. Escamilla, Ashwinikumar Kulkarni, Ashley G. Anderson, Matthew Harper, Noriyoshi Usui, Jacob Ellegood, Jason P. Lerch, Shari G. Birnbaum, Haley O. Tucker, Craig M. Powell, and Genevieve Konopka, which appeared on pages 10917-10931 of the November 8, 2017 issue, the authors accidentally cited the wrong reference in the Materials and Methods section, right-hand column, line 4, on page 10918, citing Gong et al. (2003) for the Exm1.Cre mice. The correct reference should have been Gorski et al. (2002), as stated in the main body of the article on page 10918, left-hand column, last paragraph, line 3, when referring to these mice. This correction does not affect the conclusions of the article. The full reference citation has been included in the online PDF version.

DOI:10.1523/JNEUROSCI.0293-18.2018

\section{Correction: Abdelfattah et al., “A Bright and Fast Red Fluorescent Protein Voltage Indicator That Reports Neuronal Activity in Organotypic Brain Slices"}

In the article "A Bright and Fast Red Fluorescent Protein Voltage Indicator That Reports Neuronal Activity in Organotypic Brain Slices” by Ahmed S. Abdelfattah, Samouil L. Farhi, Yongxin Zhao, Daan Brinks, Peng Zou, Araya Ruangkittisakul, Jelena Platisa, Vincent A. Pieribone, Klaus Ballanyi, Adam E. Cohen, and Robert E. Campbell, which appeared on pages 2458-2472 of the February 24,2016 issue, the authors regrettably note that the units on the $y$-axes of Figure $3, D$ and $E$, were incorrectly labeled as $\Delta F / F_{0}$. The $y$-axes should instead be labeled as $F / F_{\min }$. Figure 3, $D$ and $E$, has now been replaced with the correct image in the online PDF version and is displayed below. The figure legend has been revised to include definitions of $\Delta F / F_{0}$ and $F / F_{\min }$. This correction does not affect the conclusions of the article. 
A
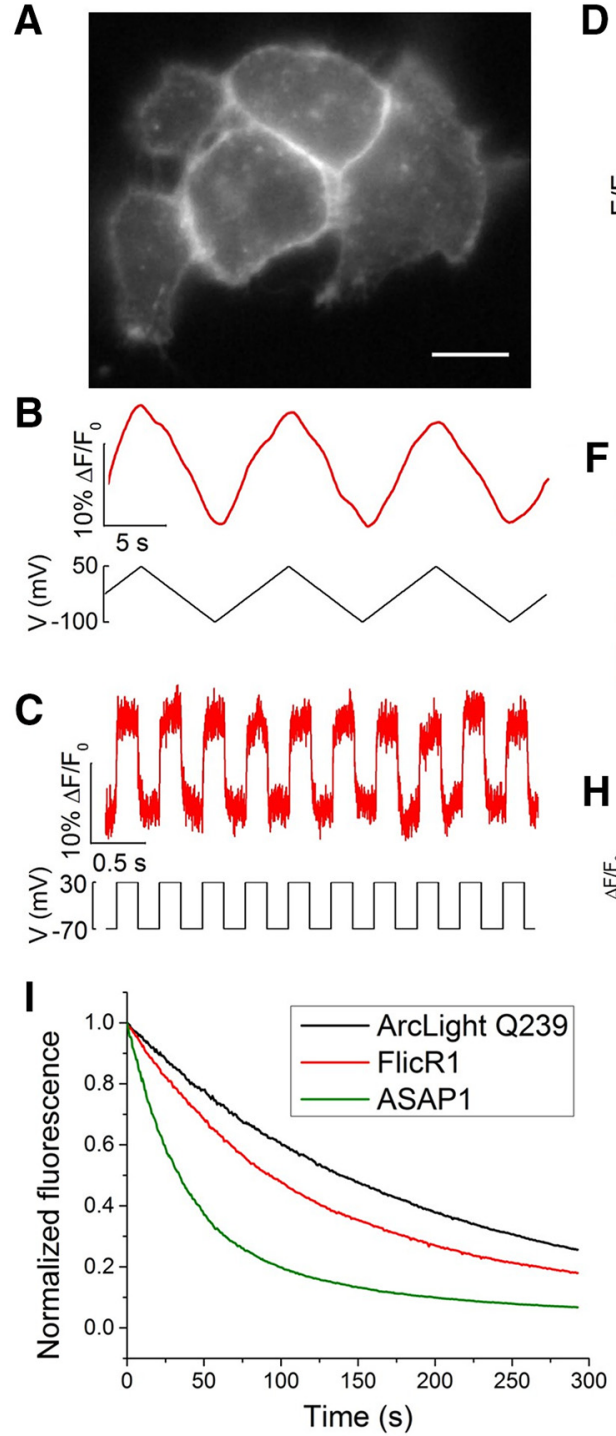

D

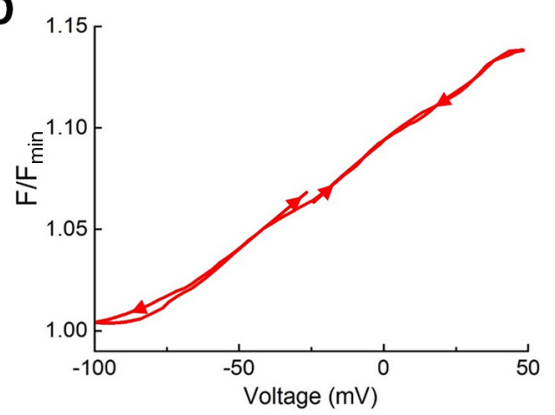

$\mathbf{F}$

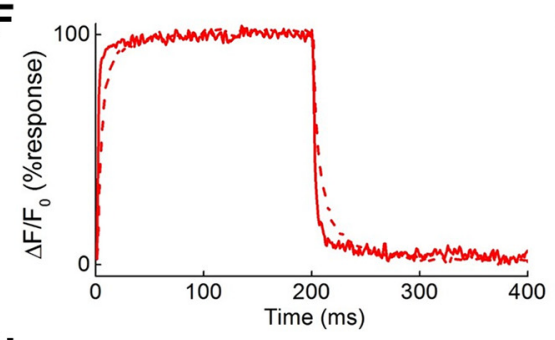

H

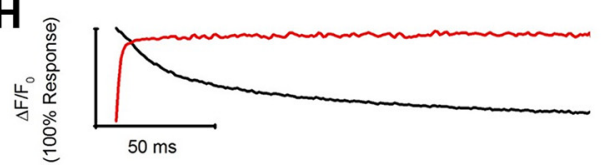

E
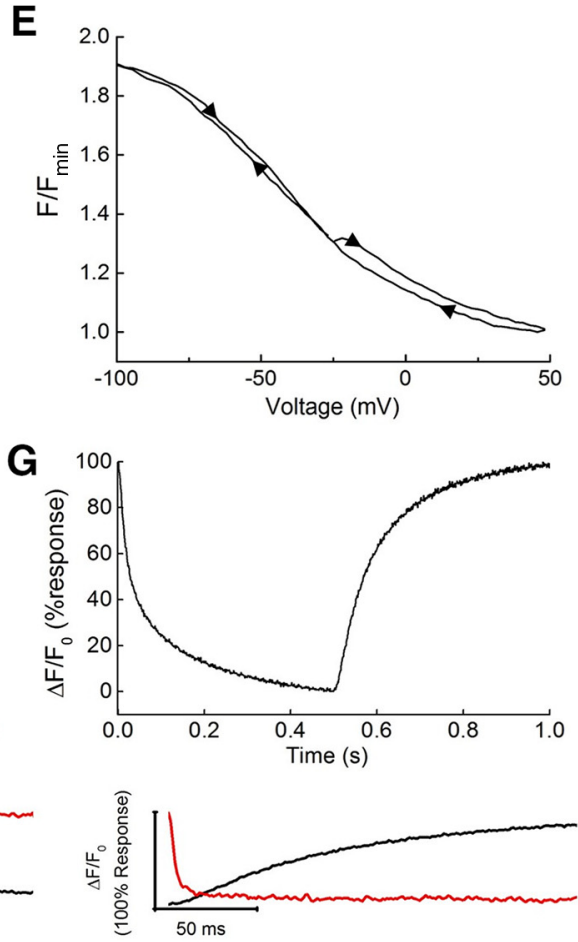
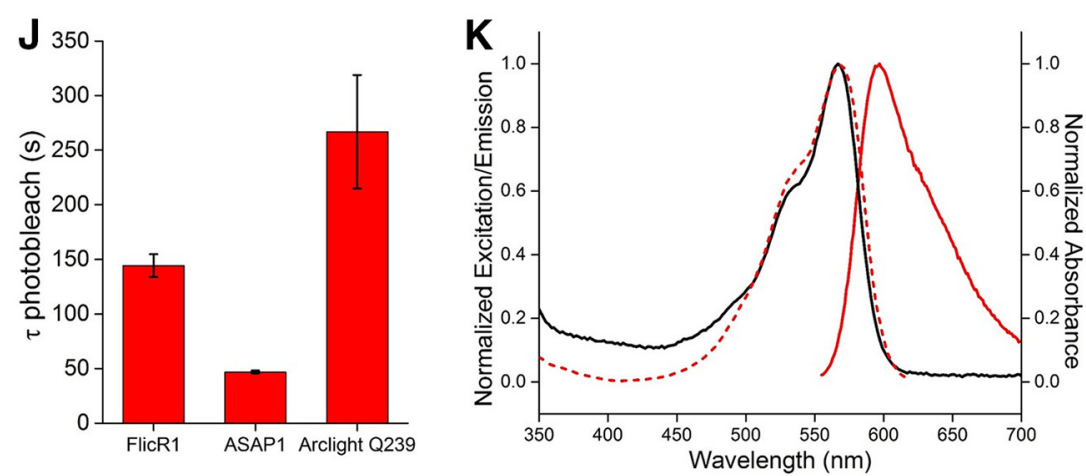

Figure 3. Characterization of FlicR1. A, Image of HEK293 cells expressing FlicR1 under the CMV promoter. Scale bar, $10 \mu \mathrm{m}$. $\boldsymbol{B}$, Fluorescence response (top) to a triangle wave in membrane potential (bottom) from $-100 \mathrm{mV}$ to $+50 \mathrm{mV}$. Fluorescence trace (acquired at $10 \mathrm{~Hz}$ ) is filtered using a 15 point moving-average low-pass filter. $\Delta F / F_{0}$ is calculated as $\left(F-F_{0}\right) / F_{0}$, where $F$ is the fluorescence at a particular time point and $F_{0}$ is initial fluorescence. C, FlicR1 fluorescence response (top) from a representative cell to a square wave in membrane potential (bottom) from $-70 \mathrm{mV}$ to $+30 \mathrm{mV}$. D, $\boldsymbol{E}$, FlicR1 (D) and Arclight Q239 (E) fluorescence as a function of membrane voltage in a representative HEK293 cell. Fluorescence $\left(F / F_{\text {min }^{\prime}}\right.$ where $F$ is the fluorescence intensity at a particular membrane voltage and $F_{\min }$ is the minimum fluorescence intensity over the range of $-100 \mathrm{mV}$ to $+50 \mathrm{mV}$ ) is the mean of three ramp cycles from $-100 \mathrm{mV}$ to $+50 \mathrm{mV}$ and back. Fluorescence is plotted starting at $-25 \mathrm{mV}$, depolarizing to $+50 \mathrm{mV}$, hyperpolarizing to $-100 \mathrm{mV}$, and then returning back up to $-25 \mathrm{mV}$, as marked by the arrows. Fluorescence showed little hysteresis between increasing and decreasing voltage ramps. $F$, FlicR1 fluorescence response to a $100 \mathrm{mV}$ step potential in HEK2 23 cells. Solid line shows fluorescence response at $34^{\circ} \mathrm{C}$. Dotted line shows fluorescence response at $22^{\circ} \mathrm{C}$. G, ArcLight Q239 fluorescence response to a $100 \mathrm{mV}$ step potential in HEK293 cells at $22^{\circ} \mathrm{C}$. Note the different time axis compared with $\boldsymbol{F}$. $\boldsymbol{H}$, Magnification of the "on" and "off" portions of $22^{\circ} \mathrm{C}$ fluorescence traces from FlicR (red) and Arclight (black). $I$, Normalized bleaching curves for FlicR1, ArcLight, and ASAP1 in HEK239 cells. J, Time constants for photobleaching of FlicR1, ASAP1, and ArcLight Q239 in HEK293 cells using continuous $10 \mathrm{~W} / \mathrm{cm}^{2} 561 \mathrm{~nm}$ light illumination for FlicR1 and continuous $10 \mathrm{~W} / \mathrm{cm}^{2} 488 \mathrm{~nm}$ light illumination for ASAP1 and ArcLight Q239. Fluorescence was captured every $500 \mathrm{~ms}$. Time constants are based on single exponential fits. Error bars indicate SEM for FlicR1 ( $n=5$ cells), ASAP1 ( $n=5$ cells), and ArcLight Q239 ( $n=4$ cells). $\boldsymbol{K}$, Spectral characterization of FlicR1 in vitro. Shown are absorbance (solid black line), excitation (dotted red line), and emission (solid red line) of FlicR1. Fluorescence imaging for voltage sensitivity measurements was performed at $10 \mathrm{~Hz}$. Step responses were recorded at $2 \mathrm{kHz}$ for FlicR1 and $1 \mathrm{kHz}$ for ArcLight Q239. Illumination intensities were $10 \mathrm{~W} / \mathrm{cm}^{2}$. 